\title{
ON THE NUMBER OF ABSOLUTE POINTS OF A CORRELATION
}

\author{
A. J. Hoffman, M. Newman, E. G. Straus \\ AND O. TAUSSKY
}

1. Introduction. In 1948, R. W. Ball [2] presented methods for obtaining information about the number of absolute points of a correlation of a finite projective plane in which neither the theorem of Desargues nor any other special property (except, of course, the existence of the correlation) is assumed. This work was, in a sense, a continuation of an earlier investigation by $R$. Baer [1] of the case that the correlation is a polarity.

We shall show how, using an incidence-matrix approach ${ }^{1}$, one may obtain the principal results of [2] somewhat more directly. Some of the results are strengthened. In addition, our method is sufficiently general to apply at once to the so-called symmetric group divisible designs, a class of combinatorial configurations including the finite projective planes. For simplicity, we shall present our main discussion in the language of planes, reserving to the end indications of the generalization.

As pointed out in $\S \S 3$ and 4 the geometric problem with which we are concerned leads naturally to the question: What are the irreducible polynomials whose roots are roots of natural numbers? This question is treated in the following section.

2. Polynomials whose roots are roots of natural numbers. Let $f(x)$ be an irreducible polynomial with integral coefficients and let one of its roots be $z=n^{1 / k} \zeta$, ( $n, k$ natural numbers, $\zeta$ a root of unity). Clearly $z$ satisfies the equation

$$
z^{k} / n=\zeta^{k}=\zeta_{h}
$$

for some $h$, where from now on we use $\zeta_{h}$ to denote a primitive $h$ th root of unity. From (1) we see that $\Phi_{h}\left(z^{k} / n\right)=0$, where $\Phi_{h}$ is the cyclotomic polynomial of order $h$. Hence

$$
f(x) \mid n^{\varphi(h)} \Phi_{h}\left(x^{k} / n\right) .
$$

The problem is therefore reduced to that of finding the irreducible factors of $\Phi_{h}\left(x^{k} / n\right)$ for arbitrary positive integers $h, k, n$. It will suffice

Received August 16, 1954. The work of the first two authors was supported (in part) by the Office of Naval Research.

1 Arithmetic properties of the incidence matrix have been exploited with conspicuous success $([\mathbf{4}],[\mathbf{5}])$. In this paper we study its characteristic polynomial. 
for our purpose here to consider only the reducibility of $\Phi_{h}\left(x^{2} / n\right)$ (that is the case $k=2$ ). The general case is settled in the note following this paper [9].

If $n^{\varphi(h)} \Phi_{h}\left(x^{2} / n\right)$ is divisible by an irreducible polynomial $g(x)$, then $g(x)$ is not a polynomial in $x^{2}$. Hence $g(-x)$, which also divides $n^{\varphi(h)} \Phi_{h}\left(x^{2} / n\right)$, is different from $g(x)$ and is irreducible. Therefore,

$$
n^{\varphi(h)} \Phi_{h}\left(x^{2} / n\right)= \pm g(x) g(-x),
$$

for $g(x) g(-x)$ is a polynomial in $x^{2}$, and $n^{৫(h)} \Phi_{h}\left(x^{2} / n\right)$ is irreducible in $x^{2}$. Then by (3), $\sqrt{ } n \zeta_{h}$ or $-\sqrt{ } n \zeta_{h}$ is a root of $g(x)$; thus $\zeta_{h}=\left( \pm \sqrt{ } n \zeta_{h}\right)^{2} / n$ is in the splitting field for $g(x)$. Thus the splitting field for $g(x)$ contains the $h$ th roots of unity; but by (3), the degree of this splitting field is $\varphi(h)$. Therefore the splitting field for $g(x)$ is the same as $R\left(\zeta_{h}\right)$. Conversely since $\sqrt{n} \zeta_{h}$ is a root of $\Phi_{h}\left(x^{2} / n\right), \sqrt{n} \zeta_{h} \in R\left(\zeta_{h}\right)$ implies that $\Phi_{h}\left(x^{2} / n\right)$ is reducible. We are thus led to the following lemma:

LEMma 1. The polynomial $\Phi_{h}\left(x^{2} / n\right)$ is reducible if and only if $\sqrt{ } n \zeta_{h}$ is contained in $R\left(\zeta_{n}\right)$.

LEMmA 2. The polynomial $\Phi_{n}\left(x^{2} / n\right)$ where $n=n^{* 2} n^{\prime}, n^{\prime}$ squarefree, is reducible if and only if $n^{\prime} \mid h$ and one of the following conditions holds:

(a) $h \equiv 1(\bmod 2)$ and $n^{\prime} \equiv 1(\bmod 4)$;

(b) $h \equiv 2(\bmod 4)$ and $n^{\prime} \equiv 3(\bmod 4)$;

(c) $h \equiv 4(\bmod 8)$ and $n^{\prime} \equiv 0(\bmod 2)$.

Proof. We first list for convenience several facts to which we shall make reference in the course of this proof and subsequently.

(i) The discriminant of a subfield of an algebraic number field divides the discriminant of the whole field [7, p. 95, Satz 39].

(ii) The discriminant of $R(\sqrt{m}), m$ a squarefree integer, is $4 m$ if $m \equiv 2,3(\bmod 4)$, and $m$ if $m \equiv 1(\bmod 4)$ [7, p. 157, Satz 95].

(iii) The discriminant of the field of the $m$ th roots of unity is divisible only by primes which divide $m$ [7, p. 146, Satz 88].

$$
\sum_{j=0}^{m-1} \zeta_{m}^{j^{2}}=\left\{\begin{array}{lll}
(1+i) \sqrt{m} & \text { if } & m \equiv 0(\bmod 4) \\
\sqrt{m} & \text { if } & m \equiv 1(\bmod 4) \\
i \sqrt{m} & \text { if } & m \equiv 3(\bmod 4)
\end{array}\right.
$$

[8, p. 177, Theorem 99].

(v) If $(r, s)=1$, then $\zeta_{r} \zeta_{s}$ is a primitive $r s$ th root of unity.

(vi) If $m$ is odd and squarefree, $m \mid r$ then $\left\{(-1)^{(m-1) / 2} m\right\}^{1 / 2} \in R\left(\zeta_{r}\right)$ (This can be shown in a variety of ways: for example, from (iv) or from (i), (ii), (iii)). 
We now turn to the proof proper. We first prove the necessity. Assume $\Phi_{h}\left(x^{2} / n\right)$ is reducible; that is, by Lemma 1 ,

$$
\sqrt{n^{\prime} \zeta_{h}} \in R\left(\zeta_{h}\right)
$$

Therefore $\sqrt{n^{\prime}} \in R\left(\sqrt{\zeta_{h}}\right)$, so by (i), (ii), (iii), $n^{\prime}$ is the product of primes each of which divides $2 h$. If $h$ is even, then $n^{\prime} \mid h$. If $h$ is odd, then since $\varphi(2 h)=\varphi(h)$, we have $R\left(v^{\prime} \zeta_{h}\right)=R\left(\zeta_{h}\right)$, so that by (i), (ii) and (iii) we have again $n^{\prime} \mid h$. Next,

(a) Assume $h$ odd. Then $\sqrt{\zeta_{h}} \in R\left(\zeta_{h}\right)$, so that (4) implies $n^{\prime} \in R\left(\zeta_{h}\right)$. Further $n^{\prime}$ is odd, since $n^{\prime} \mid h$, so either $n^{\prime} \equiv 1(\bmod 4)$ or $n^{\prime} \equiv 3(\bmod 4)$. But we cannot have $n^{\prime} \equiv 3(\bmod 4)$, for, by (ii), (i), and (iii), this would imply $2 \mid h$.

(b) Assume $h \equiv 2(\bmod 4)$. Then, since $\varphi(2 h)>\varphi(h)$, it follows that $\sqrt{\zeta_{h}} \notin R\left(\zeta_{h}\right)$, so $\sqrt{n^{\prime} \zeta_{h}} \in R\left(\zeta_{h}\right)$ implies $\sqrt{n^{\prime}} \notin R\left(\zeta_{h}\right)$. If $n^{\prime}$ is odd, this implies $n^{\prime} \equiv 3(\bmod 4)$, by the fact that $n^{\prime} \mid h$ and (vi). Further $n^{\prime}$ cannot be even. If $n^{\prime}$ were even, write $n^{\prime}=2 n^{\prime \prime}$. There are two cases : $n^{\prime \prime} \equiv 1(\bmod 4), n^{\prime \prime} \equiv 3(\bmod 4)$. If $n^{\prime \prime} \equiv 1(\bmod 4)$, then $\sqrt{n^{\prime} \zeta_{h}}=$ $\checkmark 2 \sqrt{ } n^{\prime \prime} \sqrt{\zeta_{h}} \in R\left(\zeta_{h}\right)$ implies $\sqrt{2} \sqrt{\zeta_{h}} \in R\left(\zeta_{h}\right)$, since $\sqrt{n^{\prime \prime}} \in R\left(\zeta_{h}\right)$ by the fact that $n^{\prime \prime} \mid h$ and (vi). But this means $V_{2} \in R\left(\zeta_{h}\right)$, which is impossible. For $R\left(\sqrt{\zeta_{h}}\right)$ contains $i$; and if it also contains $\sqrt{\mathbf{2}}$, it would contain $\zeta_{8}=(1+i) / \sqrt{ } 2$. By $(\mathrm{v})$, it follows that $R\left(\sqrt{\zeta_{h}}\right)$ would then contain a primitive $8(h / 2)=4 h$ th root of unity; therefore the degree of $R\left(\zeta_{h}\right)$ would be at least $\varphi(4 h)>\varphi(2 h)$; the actual degree of $R\left(\sqrt{\zeta_{h}}\right)$.

If $n^{\prime \prime} \equiv 3(\bmod 4)$, then $\sqrt{n^{\prime \prime} \zeta_{h}} \in R\left(\zeta_{h}\right)$, for $i \sqrt{n^{\prime \prime}} \in R\left(\zeta_{h}\right)$ by the fact that $n^{\prime \prime} \mid h$ and (vi), and it is easy to see (for example by (v)) that $i \sqrt{\zeta_{h}} \in R\left(\zeta_{h}\right)$. Therefere $V n^{\prime \prime} \zeta_{h}=\left(i \sqrt{ } n^{\prime \prime}\right)\left(-i \sqrt{\zeta_{h}}\right) \in R\left(\zeta_{h}\right)$. Hence, $\sqrt{2} \in R\left(\zeta_{h}\right)$, and a fortiori $\sqrt{2} \in R\left(\sqrt{\zeta_{h}}\right)$, and the preceding argument applies.

(c) Assume finally $h \equiv 4(\bmod 8)$. Then $n^{\prime}$ cannot be odd. For since $R\left(\zeta_{h}\right)$ contains $i$, and $n^{\prime} \mid h$, we learn from (vi) that $\sqrt{n^{\prime}} \in R\left(\zeta_{h}\right)$.

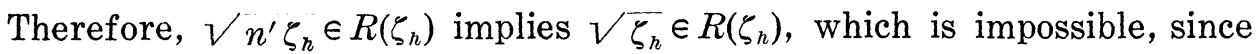
$\varphi(2 h)>\varphi(h)$.

It remains to show that if $h \equiv 0(\bmod 8)$, then $V n^{\prime} \zeta_{h} \notin R\left(\zeta_{h}\right)$ for any $n^{\prime}$. The argument used in (c) shows that $n^{\prime}$ cannot be odd. If $n^{\prime}$ were even, $n^{\prime}=2 n^{\prime \prime}$, then since $\zeta_{3}=(1+i) / \sqrt{2}$ and $i \in R\left(\zeta_{h}\right)$, we have $\sqrt{2} \in R\left(\zeta_{h}\right)$. Hence $V n^{\prime} \zeta_{h}=\sqrt{ } 2 n^{\prime \prime} \zeta_{h} \in R\left(\zeta_{h}\right)$ implies $V n^{\prime \prime} \zeta_{h} \in R\left(\zeta_{h}\right)$. Then we may use the argument just given to cover the case in which $n^{\prime}$ is odd. Hence we cannot have $h \equiv 0(\bmod 8)$.

The sufficiency is established simply by constructing $g(x)$ of (3). We first prove that in cases (a), (b)

$$
z=n^{*} \zeta_{h} \sum_{j=0}^{n{ }^{\prime}-1} \zeta_{h}^{h j^{2} / n^{\prime}}=\frac{n^{*} n^{\prime}}{h} \zeta_{h} \sum_{j=0}^{n-1} \zeta_{h}^{h j^{2} / n^{\prime}}
$$


is a zero of $\Phi_{h}\left(x^{2} / n\right)$. Since $\zeta_{h}^{h / n^{\prime}}$ is a primitive $n^{\prime}$ th root of unity we obtain from (iv):

$$
\begin{aligned}
& \text { in case (a) } z=n^{*} \sqrt{n^{\prime}} \zeta_{n}=\sqrt{n} \zeta_{h}=\mathrm{a} \text { zero of } \Phi_{h}\left(x^{2} / n\right) ; \\
& \text { in case (b) } z=n^{*} \sqrt{n^{\prime} i \zeta_{h}}=\sqrt{n} \zeta_{k} \zeta_{h}=\text { a zero of } \Phi_{h}\left(x^{2} / n\right) .
\end{aligned}
$$

In case (c) we have

$$
z=\frac{1}{2} n^{*} \zeta_{h} \sum_{j=\mathrm{J}}^{2 n^{\prime}-1} \zeta_{h}^{h j^{2} \mid I n^{\prime}}=\frac{1}{2} n^{*} \sqrt{2 n^{\prime}}(1+i) \zeta_{h}=n^{*} \sqrt{n^{\prime} \zeta_{8} \zeta_{h}}
$$

a zero of , $\Phi_{n}\left(x^{2} / n\right)$. The conjugates $z^{(l)}$ of $z$ in $R\left(\zeta_{h}\right)$ are now obtained simply by substituting $\zeta_{h}^{l}$ for $\zeta_{h}$ in (5) or $(6)$ where $(l, h)=1$. Thus we obtain

$$
g(x)=\prod_{\substack{l=1 \\(l, h)=1}}^{h-1}\left(x-z^{(l)}\right)
$$

Later on we shall need the sum of the $z^{(l)}$. We therefore establish the following lemma:

LEMma 3. If (3) holds, then the sum of the roots of $g(x)$ is

(a) $\pm n^{*} n^{\prime}$ if $h \neq 0(\bmod 4)$ and squarefree,

(b) 0 if $h \neq 0(\bmod 4)$ and $h$ is not squarefree,

(c) $\pm n^{*} n^{\prime}$ if $h \equiv 0(\bmod 4)$ and $h / 4$ is odd and squarefree,

(d) 0 if $h \equiv 0(\bmod 4)$ and $h / 4$ is odd and not squarefree.

Proof. Let us first note that, by Lemma 2, the foregoing enumeration accounts for all cases in which $n^{\varphi(h)} \Phi_{h}\left(x^{2} / n\right)$ may be reducible. Also, the \pm in (a) and (c) is to be expected, since we are clearly unable to distinguish between $g(x)$ and $g(-x)$.

We now set $h=2^{e} p_{1}{ }^{e_{1}} \cdots p_{k}^{e_{k}}, n^{\prime}=2^{\varepsilon} p_{1}^{\varepsilon_{1}} \cdots p_{k}^{\varepsilon} k$ where $\varepsilon, \varepsilon_{i}=0,1$; and write $h_{\lrcorner}=2^{e}, h_{i}=p_{i}^{e_{i}} ; n_{0}^{\prime}=2^{\varepsilon}, n_{i}^{\prime}=p_{i}^{\varepsilon_{i}} ; \zeta_{(0)}=\zeta_{h_{\jmath}}, \zeta_{(j)}=\zeta_{h_{j}}$.

Then $\zeta_{h}=\zeta_{(0)} \zeta_{(1)} \cdots \zeta_{(k)}$ and its conjugates $\zeta_{h}^{l}$ are the products of the conjugates $\zeta_{(0)}^{l_{0}}, \zeta_{(1)}^{l_{1}}, \cdots, \zeta_{(k)}^{l} k$ where $l=l_{i}\left(\bmod h_{i}\right)$. Cases $(\mathrm{a}),(\mathrm{b})$ of this lemma correspond to cases (a), (b) of Lemma 2. Here $\zeta_{0}= \pm 1$ so that we obtain from (5)

$$
\sum z^{(l)}= \pm n^{*} \sum_{j=0}^{n^{\prime}-1} \prod_{i=1}^{k} \sum_{\substack{l_{i}=1 \\\left(l_{i}, p_{i}\right)=1}}^{n_{i}-1} \zeta_{\substack{i \\(i)}}^{l_{i}\left[n j^{2} / n^{\prime}+1\right]} .
$$

As $j$ runs from 0 to $n^{\prime}-1$ its residues $\left(\bmod n_{2}^{\prime}\right)$ run independently from 0 to $n_{i}^{\prime}-1$; hence we can write 


$$
a=\sum z^{(l)}= \pm n^{*} \prod_{i=1}^{k} \sum_{j_{i}=0}^{n_{i}^{\prime}-1} \sum_{\substack{i \\\left(l_{i}, p_{i}\right)=1}}^{n_{i}-1} \zeta_{(i)}^{l_{i}\left[h_{i}{ }^{2} / n^{\prime}+1\right]}= \pm n^{*} a_{1} \cdots a_{k}
$$

In order to evaluate the $a_{i}$ we first observe that the sum of the primitive $m$ th roots of unity

$$
\sum_{\substack{l=1 \\(l, m)=1}}^{m-1} \zeta_{m}^{l}=\mu(m)
$$

This is seen most simply by observing that

$$
\Phi_{m}(x)=\prod_{d i m}\left(x^{a}-1\right)^{\mu(m / d)}=x^{\varphi(m)}-\mu(m) x^{\varphi(m)-1}+\cdots .
$$

Now for $h_{i}>n_{i}^{\prime}$ we have $\zeta_{(i)}^{\left[h_{j}^{2} / n^{\prime}+1\right]}$ a primitive $h_{i}$ th root of unity and therefore

$$
a_{i}=\sum_{j_{i}=0}^{n_{i}^{\prime}-1} \sum_{\substack{i \\\left(l_{i}=1 \\ l_{i}, p_{i}\right)=1}}^{n_{i}-1} \zeta_{(i)}^{l}\left[h_{i}{ }^{2} / n^{\prime}+1\right]=\sum_{j_{i}=0}^{n^{\prime}-1} \mu\left(h_{i}\right)=n_{i}^{\prime} \mu\left(h_{i}\right) .
$$

For $h_{i}=n_{i}^{\prime}$ we have $h / n^{\prime}$ relatively prime to $p_{i}$ so that

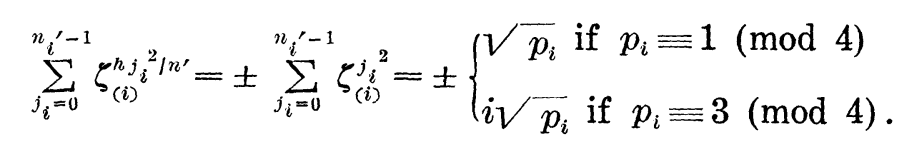

Where the sign depends on whether $h / n^{\prime}$ is or is not a quadratic residue $\left(\bmod p_{i}\right)$. Similarly

$$
\sum_{j_{i}=0}^{n_{i}^{\prime}-1} \zeta_{(i)}^{l_{i} j_{i}{ }^{2}}=\left(\frac{l_{i}}{p_{i}}\right)^{n_{i_{i}}{ }^{\prime-1}} \sum_{j^{\prime}=0}^{\zeta_{(i)}{ }^{2}}, \quad\left(\frac{l_{i}}{p_{i}}\right)=\text { Legendre symbol. }
$$

From (11) and (12) we obtain

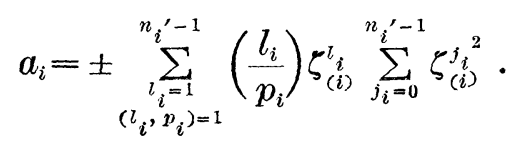

Now

$$
\sum\left(\frac{l_{i}}{p_{i}}\right) \zeta_{(i)}^{l}=\sum_{1} \zeta_{(i)}^{s}-\sum_{2} \zeta_{(i)}^{t}
$$

where $\Sigma_{1}$ ranges over those $s$ in $1, \cdots, p_{i}-1$ which are quadratic residues $\left(\bmod p_{i}\right)$ and $\sum_{2}$ ranges over those $t$ in $1, \cdots, p_{i}-1$, which are quadratic nonresidues $\left(\bmod p_{i}\right)$. According to $(9)$

$$
\sum_{1} \zeta_{(i)}^{s}+\sum_{1} \zeta_{(i)}^{t}=\mu\left(p_{i}\right)=-1
$$


and obviously

$$
\sum_{j_{i}=0}^{n_{i}^{\prime}-1} \zeta_{(i)}^{j_{i}^{2}}=1+2 \sum_{1} \zeta_{(i)}^{s}
$$

Combining (15) and (16) we have

$$
\sum_{1} \zeta_{(i)}^{s}-\sum_{2} \zeta_{(i)}^{t}=\sum_{j_{i}=0}^{n_{i}^{\prime}-1} \zeta_{(i)}^{j_{i}{ }^{2}}
$$

Substitution in (13) now yields

$$
a_{i}= \pm\left(\sum_{j_{i}=0}^{n_{i}^{\prime}-1} \zeta_{(i)}^{j_{i}{ }^{2}}\right)^{2}= \pm p_{i}=\mp n_{i}^{\prime} \mu\left(h_{i}\right) .
$$

From (8), (10) and (18) we now obtain

$$
a= \pm n^{*} n^{\prime} \mu(h)
$$

which proves cases (a), (b). In cases (c), (d) we have case (c) of Lemma (2) and therefore equation (6) obtains. We now have $a= \pm n^{*} a_{i} a_{1} \cdots a_{k}$ where $a_{1}, \cdots, a_{k}$ are the same as in (10) and (18). The only new factor is according to (6)

$$
a_{\lrcorner}=\frac{1}{2} \sum_{\substack{l_{0}=1 \\ l_{0} \text { odd }}}^{3} \sum_{j_{0}=0}^{3} \zeta_{(0)}^{l_{0}\left[h_{0} j_{0}{ }^{2} / 4+1\right]} .
$$

If $h_{\mathrm{J}}>4$ then, as in (10), we obtain

$$
a_{0}=2 \mu\left(h_{0}\right)=n_{0} \mu\left(h_{0}\right)=n_{0} \mu\left(h_{0} / 2\right)=0 .
$$

If $h_{0}=4$ then $\zeta_{(0)}=i$ and

$$
a_{v}=\frac{1}{2}\left[\zeta_{(0)}+\zeta_{(0)}^{2}+\zeta_{(0)}^{5}+\zeta_{(0)}^{10}+\zeta_{(0)}^{3}+\zeta_{(0)}^{6}+\zeta_{(0)}^{15}+\zeta_{(0)}^{30}\right]=-2=n_{0} \mu\left(h_{0} / 2\right) .
$$

Thus, finally, in cases (c), (d)

$$
a= \pm n^{*} n^{\prime} \mu(h / 2)
$$

which proves these cases.

3. The incidence matrix. We assume that we have a finite projective plane $\Pi$ with $n+1$ points on a line, $n>1$, and consequently $N=n^{2}+n+1$ points in the plane. We further assume that the plane admits a correlation $\rho$, that is a one-to-one mapping of the set of points of $I I$ onto the set of lines of $I I$, together with a one-to-one mapping of the set of lines of $I I$ onto the set of points of $I I$ such that a point is on a line if and only if the image of the point is on the image of the line. 
Our attack on the study of the number of absolute points of a correlation, that is, the set of points each of which lies on its image, is based on the following:

Lemma 4. Let $\rho$ be a correlation of a finite projective plane II, and let the points $P_{1}, \cdots, P_{N}$ and lines $l_{1}, \cdots, l_{N}$ of $\Pi$ be so numbered that $\rho P_{i}=l_{i}(i=1, \cdots, N)$. Let $A=\left(a_{i, j}\right)$ be a square matrix of order $N$ defined by the rule $a_{i j}=1$ if $P_{i}$ is on $l_{j}$, and 0 otherwise, and let $P=\left(p_{i j}\right)$ be a permutation matrix defined by $p_{i j}=1$ if $\rho^{2} P_{i}=P_{j}$, and 0 otherwise. Then if $A^{T}$ denotes the transpose of $A$, we have (i) $A^{T}=P A$, and (ii) the number of absolute points of $\rho$ is $\operatorname{tr} A$ (the trace of $A$ ).

Proof. The second part of the lemma is immediate. To prove (i), observe that the $(i, j)$ th element of $A^{T}$ is $1 \Leftrightarrow a_{j i}=1 \Leftrightarrow P_{j}$ is on $l_{i} \Leftrightarrow l_{j}=\rho P_{j}$ is on $\rho l_{i}=\rho^{2} P_{i}$. But from the definition of $P$, the $(i, j)$ th element of $P A$, is $1 \Leftrightarrow \rho^{2} P_{i}$ is on $l_{j}$. Hence $A^{T}=P A$.

Of course, it is also true that if $A$ is an incidence matrix of a finite projective plane, and there exists a permutation matrix $P=\left(p_{i j}\right)$ such that $A^{T}=P A$, then the mappings $P_{i} \rightarrow l_{i} ; l_{i} \rightarrow P_{j}$, where $p_{i j}=1$, define a correlation.

Because of (ii), it is clear that knowledge of the eigenvalues of $A$ will contribute to the solution of our problem. Now, $A^{P}=P A$ implies $A$ is normal. For if $A^{T}=P A$, then $A=A^{T} P^{T}$. Hence $A A^{T}=A^{T} P^{T} P A=A^{T} A$. Thus the eigenvalues of $A A^{T}$ are the squares of the moduli of the eigenvalues of $A$. But the eigenvalues of $A A^{T}$ can easily be computed from the fact that the incidence properties of a plane imply

$$
A A^{T}=n I+J
$$

where $I$ is the identity matrix and $J$ is the matrix every element of which is unity [4]. The eigenvalues of $A A^{T}$ are

$$
(n+1)^{2}, n, n, \cdots, n \text {. }
$$

But by (24), $n+1$ is an eigenvalue of $A$ with $(1,1, \cdots, 1)$ as corresponding eigenvector; hence the eigenvalues of $A$ are

$$
n+1, \sqrt{n} e^{i \alpha_{1}}, \sqrt{n} e^{i \alpha_{2}}, \cdots, \sqrt{n} e^{i \alpha_{N}-1}
$$

Let the permutation $P$ split up into cycles of length $d_{1}, d_{2}, \cdots, d_{r}$; $d_{1}+d_{2}+\cdots+d_{r}=N$. Then the eigenvalues of $P$ are the $d_{1}$ th roots of unity, the $d_{2}$ th roots of unity, $\cdots$, and the $d_{r}$ th roots of unity. If we write out these eigenvalues of $P$ as

$$
1, e^{i \theta_{1}}, e^{i \theta_{2}}, \cdots, e^{i \theta_{N-1}}
$$

then it follows from $A^{T} A^{-1}=P$, the normality of $A,(26)$, and (27) that 


$$
e^{-i \theta_{j}}=e^{2 i \alpha_{j}} \quad j=1,2, \cdots, N-1 .
$$

These elementary consideraticns alone suffice to prove the following:

Theorem 1 (see [2, Theorem 2.1] and [1, Theorem 4]). If $n=n^{* 2} n^{\prime}$, where $n^{\prime}$ is squarefree, and $M$ is the number of absolute points of $\rho$, then $M \equiv 1\left(\bmod n^{*} n^{\prime}\right)$.

Proof. By (26) and Lemma 4, we have

$$
M=n+1+\vee n t,
$$

where $t=\sum_{j=1}^{N-1} e^{i \alpha_{j}}$ is an algebraic integer, by (27) and (28). Therefore, $(M-(n+1))^{2} \equiv 0(\bmod n)$, which implies the theorem.

4. The characteristic polynomial. By virtue of (26), the characteristic polynomial of $A$ may be written

$$
(x-(n+1)) Q(x),
$$

where $Q(x)=\left(x-\sqrt{n} e^{i \alpha_{1}}\right)\left(x-\sqrt{n} e^{i \alpha_{2}}\right) \cdots\left(x-\sqrt{n} e^{i \alpha_{N-1}}\right)$. Then since $N-1$ $=n^{2}+n$ is even, we have

$$
Q(x) Q(-x)=\left(x^{2}-n e^{2 i \alpha_{1}}\right)\left(x^{2}-n e^{2 i \alpha_{2}}\right) \cdots\left(x^{2}-n e^{2 i \alpha_{N-1}}\right) .
$$

From (27), the fact that the complex conjugate of a $d$ th root of unity is a $d$ th root of unity, and the definition of $d_{1}, d_{2}, \cdots, d_{r}$, we may write the characteristic polynomial of $P$ as

$$
\prod_{i=1}^{r}\left(x^{a_{i}}-1\right)=(x-1)\left(x-e^{-i \theta_{1}}\right)\left(x-e^{-i \theta_{2}}\right) \cdots\left(x-e^{-i \theta_{N-1}}\right) .
$$

In (22), replace $x$ by $x^{2} / n$ and multiply both sides by $n^{N}$. There results

$$
\prod_{i=1}^{r}\left(x^{2 a_{i}}-n^{a_{i}}\right)=\left(x^{2}-n\right)\left(x^{2}-n e^{-i \theta_{1}}\right) \cdots\left(x^{2}-n e^{-i \theta_{N-1}}\right) .
$$

Comparing (33) and (31) we deduce

$$
\frac{1}{x^{2}-n} \prod_{i=1}^{r}\left(x^{2 a_{i}}-n^{a_{i}}\right)=Q(x) Q(-x)
$$

so that the irreducible factors of $Q(x)$ are of the type discussed in $\S 2$.

5. The number of absolute points of $\rho$. In this section we apply the results of $\S 2$ to present criteria sufficient to insure that $M=n+1$. If we write 


$$
Q(x)=x^{N-1}+a x^{N-2}+b x^{N-3}+\cdots,
$$

Then by (30), $M=n+1-a$.

We wish to prove that, under certain circumstances, $a=0$, and this will certainly hold if every irreducible factor of the left side of (34) is a polynomial in $x^{2}$. These factors are the irreducible factors of $\Phi_{h}\left(x^{2} / n\right)$, $h \mid d_{i}$, which were investigated in $\S 2$.

On the basis of Lemma 2, we can assert the following.

THEOREM 2. If, for each divisor of the orders $d_{1}, d_{2}, \cdots, d_{r}$ of the cycles of $P$, none of the conditions of Lemma 2 holds, then $M=n+1$. In particular (see [2]), $M=n+1$ if $n^{\prime}$ and $d=1 . c . m .\left\{d_{i}\right\}$ saiisfy one of the following:

(a) $n^{\prime} \nmid d$;

(b) $2 n^{\prime} \nmid d$ and $n^{\prime} \neq 1(\bmod 4)$;

(c) there exist odd primes $p$ and $q$ such that $p=q(\bmod 2 d)$ and $\left(n^{\prime} \mid p\right)\left(n^{\prime} / q\right)=-1$, where $(a / b)$ is the generalized Legendre-Jacobi symbol;

(d) $d=1,2$, or $p^{k}$, where $p$ is a prime $\equiv 3(\bmod 4), k$ a positive integer, $n^{\prime}>1$.

Proof. The principal statement is an immediate consequence of Lemma 2.

Proof of (a): Since $n^{\prime} \nmid d$ implies $n^{\prime} \nmid h$ for any $h \mid d_{i}$, the irreducibility of each $\Phi_{h}\left(x^{2} / n\right)$ follows from Lemma 2 .

Proof of (b): Assume (b) false. Then by virtue of (a), we may assume there exists a positive integer $h$ such that for some $d_{i}$ we have $n^{\prime}|h| d_{i}$, and $\Phi_{h}\left(x^{2} / n\right)$ reducible. If $h$ is odd, then we obtain the contradiction $n^{\prime} \equiv 1(\bmod 4)$ by Lemma 2 . If $h$ is even, then $n^{\prime}$ must be even, otherwise $2 n^{\prime} \mid h$. But by Lemma 2 (c), $n^{\prime}$ even implies $h \equiv 0(\bmod 8)$, hence we are forced to the contradiction $2 n^{\prime} \mid h$.

Proof of (c): We have $\left(n^{\prime} / p\right)\left(n^{\prime} / q\right)=-1$. Assume $\Phi_{h}\left(x^{2} / n\right)$ reducible for some $h \mid d$. Then if $h$ is odd, $n^{\prime} \equiv 1(\bmod 4)$, thus $\left(n^{\prime} / p\right)=\left(p / n^{\prime}\right)$, $\left(n^{\prime} / q\right)=\left(q / n^{\prime}\right)$, by the quadratic reciprocity law. Hence $-1=\left(n^{\prime} / p\right)\left(n^{\prime} / q\right)$ $=\left(p / n^{\prime}\right)\left(q / n^{\prime}\right)$. But $p \equiv q(\bmod 2 d)$ implies $p \equiv q\left(\bmod n^{\prime}\right)$, since $n^{\prime}|h| d$. Therefore $\left(p / n^{\prime}\right)=\left(q / n^{\prime}\right)$. Combined with $-1=\left(p / n^{\prime}\right)\left(q / n^{\prime}\right)$, this yields a contradiction.

Now let $h$ be even, $h \equiv 2(\bmod 4)$. Then by Lemma $2(b), n^{\prime} \equiv 3$ (mod 4). By the quadratic reciprocity law

$$
-1=\left(n^{\prime} / p\right)\left(n^{\prime} / q\right)=(-1)^{(p+q-2) / 2}
$$

implies $p+q \equiv 0(\bmod 4)$.

But $p \equiv=q(\bmod 2 d)$ implies $p-q \equiv \equiv(\bmod 4)$, since $h \mid d$. Therefore, $2 p \equiv 0(\bmod 4)$, contrary to the fact that $p$ is an odd prime. 
Finally, let $h \equiv 0(\bmod 4)$. Then by Lemma $2(\mathrm{c}), n^{\prime}$ is even. Write $n^{\prime}=2 n^{\prime \prime}$. Then

$$
-1=\left(n^{\prime} / p\right)\left(n^{\prime} / q\right)=(2 / p)(2 / q)\left(n^{\prime \prime} / p\right)\left(n^{\prime \prime} / q\right)=\left(n^{\prime \prime} / p\right)\left(n^{\prime \prime} / q\right),
$$

since $p \equiv q(\bmod 8)$. If $n^{\prime \prime} \equiv 1(\bmod 4)$, we obtain a contradiction as in the first case considered above. If $n^{\prime \prime} \equiv 3(\bmod 4)$, we obtain a contradiction as in the second case. Note that the hypothesis $p \equiv q$ $(\bmod d)($ instead of $p \equiv q(\bmod 2 d))$ is sufficient in all cases except when simultaneously $n^{\prime} \equiv 3(\bmod 4)$ and $d \not \equiv 0(\bmod 4)$.

Proof of (d): If $d=1$ (see [1, Theorem 6]) or $d=2$, then the only $h \mid d$ are $h=1$ or $h=2$. If $h=1$ we cannot have $n^{\prime} \mid h$. If $h=2$, then $n^{\prime} \mid h$ implies $n^{\prime}=2$, contrary to Lemma $2(\mathrm{~b})$. If $d=p^{k}, p$ a prime $\equiv 3(\bmod$ 4), then $h \mid d$ implies $h$ is also of this form. Assume now $\Phi_{h}\left(x^{2} / n\right)$ reducible. Since $n^{\prime} \mid h, n^{\prime}=p$. By Lemma $2(b)$, this implies $h$ is even, a contradiction.

Even in case one or more of the polynomials $n^{\varphi(h)} \Phi_{h}\left(x^{2} / n\right)$ where $h$ divides some $d_{i}$ is reducible, we may still obtain information about $M$. We can use the results of Lemma 3 as follows. Let $d_{1}, \cdots, d_{r}$ be the lengths of the disjoint cycles of $P$. For each $i=1, \cdots, r$ let $k_{i}$ be defined as follows:

(i) if $n^{\prime} \equiv 1(\bmod 4)$, let $k_{i}$ be the number of divisors of $d_{i}$ each of which is odd, squarefree and a multiple of $n^{\prime}$;

(ii) if $n^{\prime} \equiv 3(\bmod 4)$, let $k_{i}$ be the number of divisors of $d_{i}$ each of which is even, squarefree and a multiple of $n^{\prime}$;

(iii) if $n^{\prime} \equiv 2(\bmod 4)$, let $k_{i}$ be the number of divisors of $d_{i}$ each of which is a multiple of $n^{\prime}$, and of the form $4 t, t$ odd and squarefree. Then we have the following theorem.

THEOREM 3. If $k_{i}$ is defined as above, then $M=n+1+s n^{*} n^{\prime}$, where $-\sum_{i=1}^{r} k_{i} \leqq s \leqq \sum_{i=1}^{r} k_{i} . \quad$ Further, $s \equiv \sum_{i=1}^{r} k_{i}(\bmod 2)$.

Proof. All that remains to be verified is the second sentence, which follows immediately from the fact that the sum of the roots of $Q(x)$ in (34) is the sum of $\sum k_{i}$ numbers $\pm n^{*} n^{\prime}$.

6. In this section, we compare the number of absolute points of $\rho^{j}$, where $j$ is any number prime to twice the order of $\rho^{2}$, with the number of absolute points of $\rho$. The results obtained coincide with those of [2], so we shall merely sketch the present approach.

The index $j$ in what follows is an integer prime to twice the order of $\rho^{2}=2 d$. Let $M_{j}$ be the number of absolute points of $\rho^{j}$, so that $M_{1}=M$ in our previous notation. If we let $j=2 c+1$, then $P^{-c} A$ is an incidence matrix for $I I$ that bears the same relation to $\rho^{j}$ that $A$ does 
to $\rho$. In particular, $M_{j}=\operatorname{tr} P^{-c} A$. Referring back to (26), (27), and (29), we see that

$$
\begin{aligned}
& M_{1}=n+1+\sqrt{n}\left(e^{i \alpha_{1}}+\cdots+e^{i \alpha_{N}-1}\right), \\
& M_{j}=n+1+\sqrt{n}\left(e^{i j \alpha_{1}}+\cdots+e^{i j \alpha_{N}-1}\right) .
\end{aligned}
$$

But from Theorem $1, n^{-1 / 2}\left(M_{1}-(n+1)\right)$ is of the form $u \sqrt{n^{\prime}}$, where $u$ is a rational integer. Further, if $m$ is the least common multiple of the orders of the $\alpha$ 's, then $n^{-1 / 2}\left(M_{j}-(n+1)\right)$ is the image of $u \sqrt{ } n^{\prime}$ under the automorphism of $R\left(\zeta_{m}\right)$ which sends $\zeta_{m} \rightarrow \zeta_{m}^{j}$.

Now $m=d$ if $d$ is odd, $m=2 d$ if $d$ is even. In either case, however, the indices $j$ considered correspond biuniquely to all automorphisms of $R\left(\zeta_{m}\right)$. Thus, if $M_{1} \neq n+1$ (so that we know $\sqrt{n^{\prime}} \in R\left(\zeta_{m}\right)$ ), we have

$M_{j}=M_{1}$ if the automorphism $\zeta_{m} \rightarrow \zeta_{m}^{j}$ fixes $\sqrt{n^{\prime}}$,

$$
M_{j}=2(n+1)-M_{1} \text { the automorphism } \zeta_{m} \rightarrow \zeta_{m}^{j} \text { sends } \sqrt{n^{\prime}} \text { into }-\sqrt{n^{\prime}} \text {. }
$$

One may use the Gauss sums of Lemma 2 (iv) to show explicitly that in general

$$
M_{j}=\left(n^{\prime} / j\right)\left(M_{1}-(n+1)\right)+(n+1),
$$

where $\left(n^{\prime} / j\right)$ is defined to be 1 if $\left(j, n^{\prime}\right)>1$. Among other things, this formula includes the equation $M_{j}=M_{1}$ if $n$ is a square.

7. We now show how the preceding results may be extended to symmetric group divisible designs. (See [3] and [6] for a definition and discussion of the interesting properties of these designs.) For our purpose, it is appropriate to employ the following:

DEFINITION. A symmetric group divisible design $\Delta$ is a combinatorial configuration consisting of a set with $v$ elements and $v$ distinguished subsets such that

(i) each subset is incident with exactly $k$ elements, and

(ii) the subsets can be partitioned into $g$ groups, each group containing $s$ subsets $(g s=v)$, such that two distinct subsets in the same group have exactly $\lambda_{1}$ elements in common, two subsets in different groups have exactly $\lambda_{2}$ elements in common.

We assume that the design $\Delta$ admits a correlation $\rho$; that is, a one-toone mapping of the elements of $\Delta$ onto the distinguished subsets of $\Delta$, together with a one-to-one mapping of the subsets onto the elements such that an element is in a subset if and only if the image of the element contains the image of the subset. Now the existence of $\rho$ implies that in the definition given above, we may interchange, in (i) and (ii) the words subset and element. Number the elements $E_{1}, E_{2}, \cdots, E_{v}$ such 
that $E_{1}, E_{2}, \cdots, E_{s}$ are the elements of the first group, $E_{s+1}, E_{s+2}$, $\cdots, E_{2 s}$ are the elements of the second group, and so on. Number the subsets $S_{1}, S_{2}, \cdots, S_{v}$ so that $\rho E_{i}=S_{i}$. Define the incidence matrix $A=\left(a_{i j}\right)$ of order $v$, by the stipulation $a_{i j}=1$ if $E_{i}$ is in $S_{j}, 0$ otherwise, and the permutation matrix $P=\left(p_{i j}\right)$ such that $p_{i j}=1$ if and only if $\rho^{2} E_{i}=E_{j}$. Then as in the case of planes, we have

$$
\begin{aligned}
& A^{T}=P A, \text { so } A \text { is normal. Further } \\
& A A^{P}=\left(k-\lambda_{1}\right) I+\left(\lambda_{1}-\lambda_{2}\right) K+\lambda_{2} J,
\end{aligned}
$$

where $I$ and $J$ are as before, and $K$ is the direct sum of $g$ matrices of order $s$ each of which consists entirely of 1's.

Our object, as before, is to obtain a count on the number of absolute points of $\rho=\operatorname{tr} A=M$.

Since the vector $(1,1, \cdots, 1)$ is an eigenvector of $A$ and $A^{T}$ corresponding to the eigenvalue $k$, and is also an eigenvector of $K$ with eigenvalue $s$, we have from (27) that $k^{2}-\lambda_{2} v=k-\lambda_{1}+s\left(\lambda_{1}-\lambda_{2}\right)$. Hence, we may compute [1] that

$$
\left|A A^{T}-x I\right|=\left(k^{2}-x\right)\left[k+\lambda_{1}+s\left(\lambda_{1}-\lambda_{2}\right)-x\right]^{g-1}\left(k-\lambda_{1}-x\right)^{v-g} .
$$

Henceforth, let us assume $v>g>1$. This is no restriction for the combinatorial configurations apparently so excluded are realized by allowing $\lambda_{1}=\lambda_{2}$. (Indeed, the case $\lambda_{1}=\lambda_{2}$ with the further trivial restrictions $v>k>\lambda_{1}=\lambda_{2}>0$ is an important class of designs known as balanced symmetric incomplete block designs. Further, $\lambda_{1}=\lambda_{2}=1$ characterizes finite projective planes.)

Because $A$ is normal, the eigenvalues of $A A^{T}$ are the squares of the moduli of the eigenvalues of $A$. Hence, by (37), the eigenvalues of $A$ are

$$
k, \sqrt{n_{1}} e^{i \alpha_{1}}, \sqrt{n_{1}} e^{i \alpha_{2}}, \cdots, \sqrt{n_{1}} e^{i \alpha_{g-1}}, \sqrt{n_{2}} e^{i \alpha_{g}}, \cdots, \sqrt{n_{2}} e^{i \alpha_{v-1}},
$$

where $n_{1}=k-\lambda_{1}+s\left(\lambda_{1}-\lambda_{2}\right), n_{2}=k-\lambda_{1}$.

On the other hand, if $P$ is a product of disjoint cycles of lengths $d_{1}, d_{2}, \cdots, d_{r}, d_{1}+\cdots+d_{r}=v$, then the eigenvalues of $P$ are the $d_{1}$ th roots of unity, the $d_{2}$ th roots of unity, $\cdots$, the $d_{r}$ th roots of unity, namely

$$
1, e^{i \theta_{1}}, e^{i \theta_{2}}, \cdots, e^{i \theta_{v-1}} .
$$

Now by (35) and (36) we have

$$
A^{2}=\left(k-\lambda_{1}\right) P^{T}+\left(\lambda_{1}-\lambda_{2}\right) P^{T} K+\left(\lambda_{1}-\lambda_{2}\right) J .
$$

Further, each of $A, P^{T}, K, J$ commutes with the three others (for example, to check that $P^{T}$ commutes with $K$ multiply (39) on the left and right 
by $P$ and apply (35)). Hence all four of these normal matrices can be simultaneously diagonalized. Let us imagine then that (39) is in diagonal form, and examine the diagonal elements. Note that one eigenvalue of $J$ is $v$, the rest are 0 , and that $g$ eigenvalues of $K$ are $s$, the rest are 0. Clearly, then, we have

$$
\begin{aligned}
& k^{2}=\left(k-\lambda_{1}\right) \cdot 1+\left(\lambda_{1}-\lambda_{2}\right) \cdot s+\lambda_{2} \cdot v, \\
& n_{1} e^{2 i{ }_{t}}=\left(k-\lambda_{1}\right) e^{-i \theta_{j_{t}}}+\left(\lambda_{1}-\lambda_{2}\right) s e^{-i \theta_{j_{t}}}
\end{aligned}
$$

for $t=1,2, \cdots, g-1$ and some $g-1$ indices $j_{t}$ in the set $1, \cdots, v-1$, and also

$$
n_{2} e^{2 \tau \alpha} \mu_{\mu}=\left(k-\lambda_{1}\right) e^{-i \theta j_{\mu}}
$$

for $u=g, g+1, \cdots, v-1$, and $\left\{j_{\mu}\right\}$ the indices in $1,2, \cdots, v-1$ not in $\left\{j_{t}\right\}$.

We contend that the $e^{-i \theta_{j}}$ appearing in (40) can be partitioned into classes, each class consisting of a conjugate set of roots of unity. For the characteristic polynomial of $P^{T} K$ is $(x-\zeta) x^{v-g} f(x)$, where

$$
f(x)=\prod_{t-1}^{g-1}\left(x-s e^{-i \theta_{t}}\right) \text {. }
$$

But since $P^{T} K$ has rational coefficients, its characteristic polynomial is rational, hence $f(x)$ has rational coefficients. Let $h(x)=s^{\varphi(h)} \Phi_{h}(x / s)$ be the irreducible polynomial satisfied by $s e^{-i \theta j_{1}}$; that is, $e^{-i \theta_{j_{1}}}$ is a primitive $h$ th root of unity. Then $h(x)$ and $f(x)$ have a root in common, so, by the irreducibility of $h(x)$, the set of roots of $f(x)$ contains all roots of $h(x)$, namely all numbers $s \zeta_{h}$. Divide $f(x)$ by $h(x)$, apply the same argument to the quotient, and continue. This verifies our statement.

We may now imitate our previous polynomial construction in $\S 4$ for the case of planes as follows: If the characteristic polynomial of $A$ is written as $(x-k) Q(x)$ and the characteristic polynomial of $P$ as $\prod_{i=1}^{r} \Phi_{a_{i}}(x)$, then from the foregoing we have

$$
\pm Q(x) Q(-x)=n_{1}^{g-1} n_{2}^{v-g} \prod_{i} \Phi_{h_{i}}\left(x^{2} / n_{1}\right) \underset{j}{\prod_{h_{j}}}\left(x^{2} / n_{2}\right)
$$

where the $h_{i}$ and $h_{j}$ are divisors of the cycle lengths $d_{1}, d_{2}, \cdots, d_{r}$, $\sum_{i} \varphi\left(h_{i}\right)=g-1, \sum_{j} \varphi\left(h_{j}\right)=v-g$. One can then proceed from (43) by the techniques previously used in studying the consequences of (34).

\section{REFERENCES}

1. R. Baer, Polarities in finite projective planes, Bull. Amer. Math. Soc., 52 (1946), 77-93.

2. R. W. Ball, Dualities of finite projective planes, Duke. Math. J., 15 (1948), 929. 
3. R. C. Bose and W. S. Connor, Combinatorial properties of group divisible incomplete block designs, Ann. Math. Stat., 23 (1952), 367.

4. R. H. Bruck and H. J. Ryser, The nonexistence of certain finite projective planes, Canad. J. Math., 1 (1949), 88.

5. S. Chowla and H. J. Ryser, Combinatorial problems, Canad. J. Math., 2 (1950), 93.

6. W. S. Connor, Some relations among the blocks of symmetrical group divisible designs, Ann. Math. Stat., 23 (1952), 602.

7. O. Hilbert, Gesammelte Abhandlungen, Vol. 1, Berlin, 1932.

8. T. Nagell, Introduction to number theory, Uppsala, 1951.

9. E. G. Straus and O. Taussky, Remark on the preceding paper. Algebraic equations satisfied by roots of natural numbers, Pacific J. Math., 6 (1956), 000.

National Bureau of Standards

UNiversity of CALIForNia, LOS ANGElES 